\title{
对溶液法测定偶极矩实验中单位制的思考
}

\author{
许文华 ${ }^{*}$, 岳可芬*, 马洁, 张萍* \\ 西北大学化学与材料科学学院, 西安 710127
}

\begin{abstract}
摘要: 溶液法测定偶极矩是大学物理化学的一个经典实验。尽管实验操作简单, 但是数据处理相对复杂。表示实验 原理的公式基本都采用电磁学中的高斯单位制(Gaussian units), 这对于几乎只学习了国际单位制(SI)的化学背景学 生的理解额外增加了难度。本文介绍及比较了两个单位制下的实验原理的公式, 并自然得出通过实验数据计算偶极 矩的最终公式系数及其中各物理量的量纲。
\end{abstract}

关键词：偶极矩；溶液法；高斯单位制；国际单位制 中图分类号: G64; O6

\section{On the Unit Systems of Determination of the Dipole Moment in Solvents}

\author{
Wenhua Xu *, Kefen Yue ", Jie Ma, Ping Zhang * \\ College of Chemistry \& Materials Science, Northwest University. Xi'an 710127, China.
}

\begin{abstract}
Obtaining the dipole moment in solvents is a classic college-level physical chemistry experiment. The data processing is somehow tedious despite the relatively simple operations. The basic formulas are usually expressed under Gaussian units and this causes difficulties for students who are only familiar with the International System of Units (SI units) with chemistry background. Here we discuss units conversions within the scope of the experiment and emphasize the derivation of the coefficients in the final equation. This may improve both understandings and data processing of the experiment.
\end{abstract}

Key Words: Dipole moment; Solution method; Gaussian units; SI units

溶液法测定偶极矩是大学物理化学的一个经典实验。前人从实验的改进、误差的处理等方面对 该实验作了很多探讨 ${ }^{[1-9]}$ 。本实验所采用的原理(公式)来自经典电磁理论, 教材 ${ }^{[10-17]}$ 中的一系列公式 几乎都采用了高斯单位制(Gaussian units), 而最终由这些公式给出的通过实验测定计算偶极矩的公 式的量纲却是国际单位制(SI)。这种不一致给当下几乎只学习了国际单位制的本科生甚至实验教师 带来了概念上和数据处理上的困惑。尽管在电磁学领域, 由于历史传承带来的习惯和使得部分公式 形式上更简洁等多种原因, 高斯单位制仍然有着重要的意义, 但是目前的主流是采用国际单位制。 本文通过将教材中的高斯单位制的基本公式转换为SI单位制下的表示式, 从而自然地导出偶极矩在 两种单位制下、分别采用三种量纲的计算公式。 


\section{1 高斯单位制}

高斯单位制是电磁学领域中多种cgs (centimeter-gram-second)单位制的一种, 因此也被称为 Gaussian-cgs单位制。有很多文献论述了它和国际单位制的关系 ${ }^{[18-20]}$, 本文不再详细复述, 只列出 和本实验最相关的要点。首先, 不同于国际单位制引入了安培(A)作为新的基本单位(Base units), 高斯单位制没有引入新的基本单位, 因此, 诸如电流、电压、电场强度等物理量的单位可以通过 厘米(centimeter)、克(gram)和秒(second)三个基本单位导出。例如, 电量的国际单位是库伦(C), 或 者 $\mathrm{A} \cdot \mathrm{s}$, 高斯单位为 $\mathrm{esu}$ (静电单位, electrostatic unit)或者 statcoulomb (静电库伦), 用基本单位表达 为 $\left(\mathrm{cm}^{3} \cdot \mathrm{g} \cdot \mathrm{s}^{-2}\right)^{1 / 2}$ 。更多其他物理量的单位参见表 1 。

表1 本实验涉及的物理量的单位

\begin{tabular}{ccc}
\hline 物理量 & 国际单位 & 高斯单位 \\
\hline 电荷 & $\mathrm{C}$ & $\mathrm{esu}$ \\
偶极矩 & $\mathrm{C} \cdot \mathrm{m}$ & $\mathrm{esu} \cdot \mathrm{cm}$ \\
摩尔极化度 & $\mathrm{m}^{3} \cdot \mathrm{mol}^{-1}$ & $\mathrm{~cm}^{3} \cdot \mathrm{mol}^{-1}$ \\
介电常数 & 1 & 1 \\
真空电容率 & $\mathrm{C} \cdot \mathrm{V}^{-1} \cdot \mathrm{m}^{-1}$ & 1 \\
长度 & $\mathrm{m}$ & $\mathrm{cm}$ \\
质量 & $\mathrm{kg}$ & $\mathrm{g}$ \\
时间 & $\mathrm{s}$ & $\mathrm{s}$ \\
能量 & $\mathrm{J}$ & $\mathrm{Erg}$ \\
\hline
\end{tabular}

$1 \mathrm{esu}=\left(\mathrm{cm}^{3} \cdot \mathrm{g} \cdot \mathrm{s}^{-2}\right)^{1 / 2} ; 1 \mathrm{Erg}=10^{-7} \mathrm{~J}$

力学中的物理量, 例如温度、时间、质量、能量等在两种单位制下量纲一致, 均无需转换。而 电磁学的物理量, 例如电场强度 $E$ 和磁感应强度 $B$ 在高斯单位制下有着相同的量纲, 但在国际单位制 下差一个速度的量纲。由于同一物理量在两种单位制下的量纲的不同, 电磁学的某些公式也可能有 差别, 电磁学的物理量的转换可通过一定的规则完成。本实验中用到的是如下三个转换规则:

$$
\begin{aligned}
q_{\mathrm{G}} & \rightarrow \frac{q_{\mathrm{SI}}}{\sqrt{4 \pi \varepsilon_{0}}} \\
\mu_{\mathrm{G}} & \rightarrow \frac{\mu_{\mathrm{SI}}}{\sqrt{4 \pi \varepsilon_{0}}} \\
\varepsilon_{\mathrm{G}} & \rightarrow \frac{\varepsilon_{\mathrm{SI}}}{\varepsilon_{0}}
\end{aligned}
$$

$q 、 \mu 、 \varepsilon 、 \varepsilon_{0}$ 分别代表电量、偶极矩、介电常数、真空电容率(vacuum electric permittivity), G和 SI分别表示高斯单位制和国际单位制下的物理量或者公式。

\section{2 本实验中公式的转换}

\section{1 偶极矩与极化度}

偶极矩 $\mu$ 是表示分子中电荷分布情况的物理量, 其数值大小可以度量分子的极性。偶极矩是矢量, 规定其方向由正到负, 定义为分子正负电荷中心所带的电荷量 $q$ 与正负电荷中心之间的距离 $d$ 的乘积。

$$
\vec{\mu}=q \cdot \vec{d} \quad(\mathrm{G})
$$

将公式中每个符号按照上述规则替换, 消除公因子后将得到该式的SI单位下的表达式。可以容 易的验证，SI下的公式形式和高斯单位制下相同。

$$
\vec{\mu}=q \cdot \vec{d} \quad(\mathrm{SI})
$$


下面直接给出各公式的两种形式, 经常出现在实验教材中的高斯单位制下的公式在先, 转换后 的SI单位制下的公式在后。读者可以容易的验证该转换。

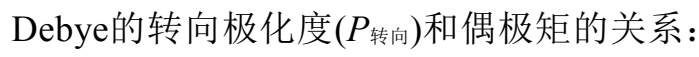

$$
\begin{aligned}
& P_{\text {转向 }}=\frac{4}{9} \pi N_{\mathrm{A}} \frac{\mu^{2}}{k T} \quad(\mathrm{G}) \\
& P_{\text {转向 }}=\frac{N_{\mathrm{A}} \mu^{2}}{9 \varepsilon_{0} k T} \quad(\mathrm{SI})
\end{aligned}
$$

其中 $k$ 为Boltzmann常数, $T$ 为热力学温度, $N_{\mathrm{A}}$ 为Avogadro常数(或用 $L$ 表示)。

Clausius-Mosotti-Debye公式的摩尔极化度 $(P)$ 与介电常数 $(\varepsilon)$ 的关系:

$$
\begin{aligned}
& P=\frac{\varepsilon-1}{\varepsilon-2} \cdot \frac{M}{\rho} \quad(\mathrm{G}) \\
& P=\frac{\varepsilon / \varepsilon_{0}-1}{\varepsilon / \varepsilon_{0}+2} \cdot \frac{M}{\rho} \quad(\mathrm{SI})
\end{aligned}
$$

注意: 不仅 SI和 G单位制下极化度的公式形式不同, 而且单位也不同, 前者单位 $\mathrm{m}^{3} \cdot \mathrm{mol}^{-1}$, 后者 单位 $\mathrm{cm}^{3} \cdot \mathrm{mol}^{-1}$ 。

\section{2 溶液法测定偶极矩}

$$
\begin{aligned}
& \varepsilon_{\mathrm{s}}=\varepsilon_{1}\left(1+\alpha x_{2}\right) \quad(\mathrm{G}) \\
& \frac{\varepsilon_{\mathrm{s}}}{\varepsilon_{0}}=\frac{\varepsilon_{1}}{\varepsilon_{0}}\left(1+\alpha x_{2}\right) \quad(\mathrm{SI}) \\
& \rho_{\mathrm{s}}=\rho_{1}\left(1+\beta x_{2}\right) \quad(\mathrm{G}, \mathrm{SI}) \\
& P_{2}^{\infty}=\frac{3 \alpha \varepsilon_{1}}{\left(\varepsilon_{1}+2\right)^{2}} \times \frac{M_{1}}{\rho_{1}}+\frac{\varepsilon_{1}-1}{\varepsilon_{1}+2} \times \frac{M_{2}-\beta M_{1}}{\rho_{1}} \quad(\mathrm{G}) \\
& P_{2}^{\infty}=\frac{3 \alpha \varepsilon_{1}}{\left(\varepsilon_{1} / \varepsilon_{0}+2\right)^{2}} \times \frac{M_{1}}{\rho_{1}}+\frac{\varepsilon_{1} / \varepsilon_{0}-1}{\varepsilon_{1} / \varepsilon_{0}+2} \times \frac{M_{2}-\beta M_{1}}{\rho_{1}} \\
& n_{\mathrm{s}}=n_{1}\left(1+\gamma x_{2}\right) \quad(\mathrm{G}, \mathrm{SI}) \\
& R_{2}^{\infty}=\frac{n_{1}^{2}-1}{n_{1}^{2}+2} \times \frac{M_{2}-\beta M_{1}}{\rho_{1}}+\frac{6 n_{1}^{2} M_{1} \gamma}{\left(n_{1}^{2}+2\right)^{2} \rho_{1}} \quad(\mathrm{G}, \mathrm{SI}) \\
& P_{\text {I }_{\text {向 }}}=P_{2}^{\infty}-R_{2}^{\infty} \quad(\mathrm{G}, \mathrm{SI}) \\
& R_{2} \text { 分别 }
\end{aligned}
$$

$P_{2}^{\infty}, R_{2}^{\infty}$ 分别是无限稀释溶液溶质的摩尔极化度和摩尔折射度, 可分别通过实验测定溶液和溶剂 的 $\varepsilon 、 n$ 和 $\rho$ (下标 $1 、 2$ 和 $\mathrm{s}$ 分别代表溶剂, 溶质和溶液)数据, 再由式(4.1)、式(5)、式(6.1)、式(7) 和式(8)求得。

\section{3 偶极矩计算公式及讨论}

\section{1 不同单位制下的偶极矩计算公式}

式(9)结合式(2.1)得,

$$
\begin{aligned}
& \mu=\sqrt{\frac{9 k T\left(P_{2}^{\infty}-R_{2}^{\infty}\right)}{4 \pi N_{\mathrm{A}}}}=\frac{3}{2} \sqrt{\frac{k}{\pi N_{\mathrm{A}}}} \cdot \sqrt{T\left(P_{2}^{\infty}-R_{2}^{\infty}\right)}=1.28 \times 10^{-20} \sqrt{T\left(P_{2}^{\infty}-R_{2}^{\infty}\right)}(\mathrm{esu} \cdot \mathrm{cm}) \\
& \text { 式(9)结合式(2.2)得, } \\
& \mu=\sqrt{\frac{9 \varepsilon_{0} k T\left(P_{2}^{\infty}-R_{2}^{\infty}\right)}{10^{6} \times N_{\mathrm{A}}}}=0.0427 \times 10^{-30} \sqrt{T\left(P_{2}^{\infty}-R_{2}^{\infty}\right)}(\mathrm{C} \cdot \mathrm{m}) \quad \text { (SI) }
\end{aligned}
$$


注意在高斯单位下, 物理常数 $k=1.380649 \times 10^{-16} \mathrm{Erg} \cdot \mathrm{K}^{-1}$, 进一步, 引入常用的偶极矩的单位 德拜 $($ Debye, $\mathrm{D}), \quad 1 \mathrm{D} \equiv 1.0 \times 10^{-18} \mathrm{esu} \cdot \mathrm{cm} \approx 3.33564 \times 10^{-30} \mathrm{C} \cdot \mathrm{m}$ ，则得，

$$
\mu=1.28 \times 10^{-2} \sqrt{T\left(P_{2}^{\infty}-R_{2}^{\infty}\right)} \text { (D) }
$$

显然, 三个不同单位的偶极矩的计算公式的系数不同; 此处我们将单位标记在后面的括号内, 与 公式中间间隔一个空格。

注意式(10.2)中的 $10^{6}$ 因子是为了 $P_{2}^{\infty} 、 R_{2}^{\infty}$ 的单位转换。这三个计算公式中 $P_{2}^{\infty} 、 R_{2}^{\infty}$ 的单位均是高斯 单位即 $\mathrm{cm}^{3} \cdot \mathrm{mol}^{-1}$ 。具体到数据处理时, $P_{2}^{\infty} 、 R_{2}^{\infty}$ 的计算完全可以采用高斯单位制, 即通过式(6.1)和 式(8), 摩尔质量、密度的单位分别为 $\mathrm{g} \cdot \mathrm{mol}^{-1}$ 和 $\mathrm{g} \cdot \mathrm{cm}^{-3}$, 这样得到的 $P_{2}^{\infty} 、 R_{2}^{\infty}$ 单位即为 $\mathrm{cm}^{3} \cdot \mathrm{mol}^{-1}$, 然后 通过 $10^{6}$ 因子将 $P_{2}^{\infty} 、 R_{2}^{\infty}$ 的单位调节为 $\mathrm{SI}$ 单位制的 $\mathrm{m}^{3} \cdot \mathrm{mol}^{-1}$ 。

本实验中涉及的物理常数参见表 $2^{[21]}$ 。

表2 本实验涉及的物理常数表

\begin{tabular}{ccccc}
\hline 物理量 & 符号 & 数值 & 单位 & 备注 \\
\hline 真空中的光速 & $c$ & 299792458 & $\mathrm{~m} \cdot \mathrm{s}^{-1}$ & 精确值 \\
Boltzmann常数 & $k$ & $1.380649 \times 10^{-23}$ & $\mathrm{~J} \cdot \mathrm{K}^{-1}$ & 精确值 \\
真空电容率 & $\varepsilon_{0}$ & $8.8541878128 \times 10^{-12}$ & $\mathrm{~F} \cdot \mathrm{m}^{-1}$ & \\
Avogadro常数 & $N_{\mathrm{A}}$ & $6.02214076 \times 10^{23}$ & $\mathrm{~mol}^{-1}$ & 精确值 \\
\hline
\end{tabular}

\section{2 教材中偶极矩计算公式讨论}

\subsection{1 关于偶极矩计算公式的系数}

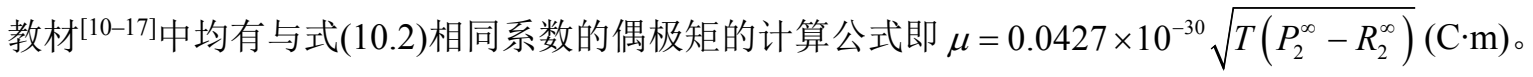
但因教材中均没有给出SI单位制下的式(3.2), 也没有出现真空电容率 $\varepsilon_{0}$, 以及不清楚公式中 $P_{2}^{\infty} 、 R_{2}^{\infty}$ 的 单位, 读者无法推得系数 $0.0426 \times 10^{-30}$ 。

笔者仅看到教材 ${ }^{[22]}$ 中有公式 $\mu=\frac{3}{2} \sqrt{\frac{k}{\pi N_{\mathrm{A}}}} \cdot \sqrt{T\left(P_{2}^{\infty}-R_{2}^{\infty}\right)}$, 该公式后未标单位, 也没有像式(10.1)那样 给出进一步的系数 $1.28 \times 10^{-20}$ 。虽教材中均给出与式(2.1)相同的公式 $P_{\text {转向 }}=\frac{4}{9} \pi N_{\mathrm{A}} \frac{\mu^{2}}{k T}$, 由此式很容易得 出上式。但由于学生不清楚是高斯单位, 以及不清楚高斯单位制下物理常数 $k=1.380649 \times 10^{-16} \mathrm{Erg} \cdot \mathrm{K}^{-1}$, 也无法推得系数 $1.28 \times 10^{-20}$ 。

教材 ${ }^{[13-16]}$ 中给出与式 $(10.3)$ 相同, 系数为 $1.28 \times 10^{-2}$ 、单位为Debye (D)的偶极矩的计算公式, 即 $\mu=1.28 \times 10^{-2} \sqrt{T\left(P_{2}^{\infty}-R_{2}^{\infty}\right)}$ (D), 但由于一方面不清楚高斯单位下物理常数 $k=1.380649 \times 10^{-16} \mathrm{Erg} \cdot \mathrm{K}^{-1}$, 另一方面不清楚偶极矩的单位德拜(Debye, D)与高斯单位的关系 $1 \mathrm{D} \equiv 1.0 \times 10^{-18} \mathrm{esu} \cdot \mathrm{cm}$, 同样无法推 得系数 $1.28 \times 10^{-2}$ 。

总之, 教材中普遍存在电磁学基本原理给出的是高斯单位制下的公式, 而最终的偶极矩计算公 式又是SI单位制下的式子。没有明确提出高斯单位制, 未介绍高斯单位制和SI单位制的转化, 内容 上前后不能很好地自洽。普遍存在对偶极矩计算公式解释不充分的情况。这显然给读者带来困惑, 因为在目前的科学教育背景下, 不加解释而默认的是国际单位制。我们将在2021年底出版的自编物 理化学实验教材第2版中对该实验原理(公式)和偶极矩计算公式加入解释。

值得注意的是, 类似的单位制不同导致公式不同的情况在大学物理化学实验 “电泳法测定 $\zeta$ 电 势” 中也存在。张小亮等对此有专门的阐述 ${ }^{[23]}$ 。 


\subsection{2 关于偶极矩计算公式的单位标记}

教材中未发现高斯单位制下偶极矩的计算公式。

偶极矩的SI单位为 $C \cdot m$, 计算公式的规范书写应如式(10.2), 单位标记在括号内, 与公式中间间 隔一个空格。同样, 偶极矩采用Debye为单位时, 计算公式的规范书写应如式(10.3), 单位标记在括 号内, 与公式中间间隔一个空格。教材存在单位的不规范甚至错误的表示, 如SI单位 C.m未写在括号 内、与公式中间未间隔一个空格或间隔了多个空格、代表单位的英文字母斜体、SI单位写成 $\mathrm{c} \cdot \mathrm{m} 。$ 这 显然容易引起读者的误解。

\section{4 结语}

本文通过将目前在大学物理化学实验教材中的 “溶液法测量偶极矩” 实验中通行的高斯单位制 下的公式转换为国际单位制下的表达, 解释了数据处理中的核心公式系数的由来。本文是对理解该 实验的一个有益甚至必须的补充。同时为理解两种单位制的差别提供了一个具体的案例。

\section{参 考 文 献}

[1] 李瑞英, 陈六平, 余小岗. 中山大学学报论丛, 2003, 23 (3), 26.

[2] Janini, G. M.; Katrib, A. H. J. Chem. Edu. 1983, 60, 1087.

[3] 江明, 丁海清, 俞马宏, 朱堂烈, 肖鹤鸣, 高怀琳. 华东工学院学报, 1992, No. 2, 85 ,

[4] 钱鹰, 丁志峰, 孙岳明, 刘举正. 东南大学学报, 1996, 26 (1), 49.

[5] 何玉葶, 李浩钧, 罗开容. 化学通报, 1989, No. 4, 54 .

[6] 孟晓燕. 江西化工, 2015, No. 6, 100 .

[7] 玉占君, 任庆云, 张婷婷. 实验技术与管理, 2007, 24 (8), 32.

[8] 史振民, 张竹莲, 巩育军, 刘生昆. 延安大学学报, 1996, 15 (1), 83.

[9] Breitung, E. M.; Vaughan, W. E.; McMahon, R. J. Rev. Sci. Instrum. 2000, 71, 224

[10] 复旦大学, 等编. 物理化学实验. 第3版. 北京: 高等教育出版社, 2004.

[11] 顾月姝, 宋淑娥. 基础化学实验(III)-物理化学实验. 第2版. 北京: 化学工业出版社, 2007.

[12] 岳可芬. 基础化学实验(III)-物理化学实验. 北京: 科学出版社. 2012.

[13] 朱万春, 张国艳, 李克昌, 徐家宁. 基础化学实验-物理化学实验分册. 第2版. 北京: 高等教育出版社, 2017.

[14] 孙尔康, 徐维清, 邱金恒. 物理化学实验. 南京: 南京大学出版社, 1998.

[15] 刘寿长, 张建民, 徐顺. 物理化学实验与技术. 郑州: 郑州大学出版社, 2004.

[16] 孟长功. 基础化学实验. 北京: 高等教育出版社, 2019.

[17] 陈六平, 戴宗. 现代化学实验与技术. 北京: 科学出版社, 2015.

[18] Garg, A. Eur. J. Phys. 2018, 39, 045205.

[19] 俞允强. 电动力学简明教程. 北京: 北京大学出版社, 1999.

[20] 尹真. 电动力学. 北京: 科学出版社, 2010.

[21] Tiesinga, E. Rev. Mod. Phys. 2021, 93 (2), 025010.

[22] 雷群芳. 中级化学实验. 北京: 科学出版社, 2005 .

[23] 汪雨, 盛寿日, 张小亮. 化学教育, 2017, 38 (10), 79. 